\title{
A SIRS Epidemic Model Incorporating Media Coverage with Random Perturbation
}

\author{
Wenbin Liu \\ College of Physics and Electronic Information Engineering, Wenzhou University, Wenzhou 325035, China \\ Correspondence should be addressed to Wenbin Liu; wenbinliu2013@163.com
}

Received 21 June 2013; Accepted 5 September 2013

Academic Editor: Yong Ren

Copyright (C) 2013 Wenbin Liu. This is an open access article distributed under the Creative Commons Attribution License, which permits unrestricted use, distribution, and reproduction in any medium, provided the original work is properly cited.

We investigate the complex dynamics of a SIRS epidemic model incorporating media coverage with random perturbation. We first deal with the boundedness and the stability of the disease-free and endemic equilibria of the deterministic model. And for the corresponding stochastic epidemic model, we prove that the endemic equilibrium of the stochastic model is asymptotically stable in the large. Furthermore, we perform some numerical examples to validate the analytical finding, and find that if the conditions of stochastic stability are not satisfied, the solution for the stochastic model will oscillate strongly around the endemic equilibrium.

\section{Introduction}

Epidemiology is the study of the spread of diseases with the objective of tracing factors that are responsible for or contribute to their occurrence. Mathematical modeling has become an important tool in analyzing the epidemiological characteristics of infectious diseases and can provide useful control measures. Various models have been used to study different aspects of diseases spreading [1-11].

Let $S(t)$ be the number of susceptible individuals, $I(t)$ the number of infective individuals, and $R(t)$ the number of removed individuals at time $t$, respectively. A general SIRS epidemic model can be formulated as

$$
\begin{gathered}
\frac{d S}{d t}=b-d S-g(I) S+\gamma R, \\
\frac{d I}{d t}=g(I) S-(d+\mu+\delta) I, \\
\frac{d R}{d t}=\mu I-(d+\gamma) R,
\end{gathered}
$$

where $b$ is the recruitment rate of the population, $d$ is the natural death rate of the population, $\mu$ is the natural recovery rate of the infective individuals, $\gamma$ is the rate at which recovered individuals lose immunity and return to the susceptible class, and $\delta$ is the disease-induced death rate. The transmission of the infection is governed by the incidence rate $g(I) S$, and $g(I)$ is called the infection force.

In modelling of communicable diseases, the incidence rate $g(I) S$ has been considered to play a key role in ensuring that the models indeed give reasonable qualitative description of the transmission dynamics of the diseases. Some factors, such as media coverage, density of population, and life style, may affect the incidence rate directly or indirectly [12-18]. It is worthy to note that, during the spreading of severe acute respiratory syndrome (SARS) from 2002 to 2004 and the outbreak of influenza A (H1N1) in 2009, media coverage plays an important role in helping both the government authority make interventions to contain the disease and people response to the disease $[12,18]$. And a number of mathematical models have been formulated to describe the impact of media coverage on the transmission dynamics of infectious diseases. Especially, Liu and Cui [15], Tchuenche et al. [17], and Sun et al. [16] incorporated a nonlinear function of the number of infective (2) in their transmission term to investigate the effects of media coverage on the transmission dynamics:

$$
g(I)=\beta_{1}-\frac{\beta_{2} I}{m+I},
$$

where $\beta_{1}$ is the contact rate before media alert; the terms $\beta_{2} I /(m+I)$ measure the effect of reduction of the contact rate when infectious individuals are reported in the media. 
Because the coverage report cannot prevent disease from spreading completely we have $\beta_{1} \geq \beta_{2}$. The half-saturation constant $m>0$ reflects the impact of media coverage on the contact transmission. The function $I /(m+I)$ is a continuous bounded function which takes into account disease saturation or psychological effects. Then model (1) becomes

$$
\begin{gathered}
\frac{d S}{d t}=b-d S-\left(\beta_{1}-\frac{\beta_{2} I}{m+I}\right) S I+\gamma R, \\
\frac{d I}{d t}=\left(\beta_{1}-\frac{\beta_{2} I}{m+I}\right) S I-(d+\mu+\delta) I, \\
\frac{d R}{d t}=\mu I-(d+\gamma) R,
\end{gathered}
$$

where all the parameters are nonnegative and have the same definitions as before.

For model (3), the basic reproduction number

$$
R_{0}=\frac{b \beta_{1}}{d(d+\mu+\delta)}
$$

is the threshold of the system for an epidemic to occur. Model (3) has a the disease-free equilibrium $P_{0}=(b / d, 0,0)$ which exists for all parameter values. And the endemic equilibrium $E^{*}=\left(S^{*}, I^{*}, R^{*}\right)$ of model (3) satisfies

$$
\begin{gathered}
b-d S-\left(\beta_{1}-\frac{\beta_{2} I}{m+I}\right) S I+\gamma R=0, \\
\left(\beta_{1}-\frac{\beta_{2} I}{m+I}\right) S I-(d+\mu+\delta) I=0, \\
\mu I-(d+\gamma) R=0
\end{gathered}
$$

which yields

$$
\begin{gathered}
S^{*}=\frac{(d+\mu+\gamma)\left(m+I^{*}\right)}{\beta_{1}\left(m+I^{*}\right)-\beta_{2} I^{*}}, \\
R^{*}=\frac{\mu I^{*}}{d+\gamma}, \\
H_{1} I^{* 2}+H_{2} I^{*}+H_{3}=0,
\end{gathered}
$$

where

$$
\begin{gathered}
H_{1}=-\frac{1}{d+\gamma}\left(\beta_{1}-\beta_{2}\right)(\gamma(d+\delta)+d(d+\mu+\delta)), \\
H_{2}=-\frac{d \beta_{1} m \mu}{d+\gamma}-\beta_{1} m(d+\delta)-b \beta_{2}+b \beta_{1}\left(1-\frac{1}{R_{0}}\right), \\
H_{3}=d m(d+\mu+\delta)\left(R_{0}-1\right) .
\end{gathered}
$$

When $R_{0}>1$, we know that $H_{1}<0, H_{3}>0$; hence, model (3) has a unique endemic equilibrium $E^{*}=\left(S^{*}, I^{*}, R^{*}\right)$. These results of model (3) were studied in [15].

On the other hand, if the environment is randomly varying, the population is subject to a continuous spectrum of disturbances $[19,20]$. That is to say, population systems are often subject to environmental noise; that is, due to environmental fluctuations, parameters involved in epidemic models are not absolute constants, and they may fluctuate around some average values. Therefore, many stochastic models for the populations have been developed and studied [21-40]. But, to our knowledge, the research on the dynamics of SIRS epidemic model incorporating media coverage with random perturbation seems rare.

In this paper, our basic approach is analogous to that of Beretta et al. [24]. They assumed that stochastic perturbations were of white noise type, which were directly proportional to distances $S(t), I(t)$, and $R(t)$ from values of $S^{*}, I^{*}$, and $R^{*}$, influenced the $S(t), I(t)$, and $R(t)$, respectively. By this method, we formulate our stochastic differential equation corresponding to model (3) as follows:

$$
\begin{gathered}
d S=b-d S-\left(\beta_{1}-\frac{\beta_{2} I}{m+I}\right) S I+\gamma R+\sigma_{1}\left(S-S^{*}\right) d B_{1}(t), \\
d I=\left(\beta_{1}-\frac{\beta_{2} I}{m+I}\right) S I-(d+\mu+\delta) I+\sigma_{2}\left(I-I^{*}\right) d B_{2}(t), \\
d R=\mu I-(d+\gamma) R+\sigma_{3}\left(R-R^{*}\right) d B_{3}(t),
\end{gathered}
$$

where $\sigma_{1}, \sigma_{2}$, and $\sigma_{3}$ are real constants and known as the intensity of environmental fluctuations; $B_{1}(t), B_{2}(t)$, and $B_{3}(t)$ are independent standard Brownian motions.

The aim of this paper is to consider the stochastic dynamics of model (8). The paper is organized as follows. In Section 2, we carry out the analysis of the dynamical properties of stochastic model (8). And in Section 3, we give some numerical examples and make a comparative analysis of the stability of the model with deterministic and stochastic environments and have some discussions.

\section{Mathematical Properties of the Deterministic Model (3)}

The following result shows that the solutions for model (3) are bounded and, hence, lie in a compact set and are continuable for all positive time.

Lemma 1. The plane $S+I+R \leq b / d$ is an invariant manifold of model (3), which is attracting in the first octant.

Proof. Summing up the three equations in (3) and denoting $N(t)=S(t)+I(t)+R(t)$, we have

$$
\frac{d N}{d t}=b-d N-\delta I \leq b-d N .
$$

Hence, by integration, we check

$$
N(t) \leq \frac{b}{d}+\left(N(0)-\frac{b}{d}\right) e^{-d t} .
$$

Hence,

$$
\lim _{t \rightarrow \infty} \sup N(t) \leq \frac{b}{d},
$$

which implies the conclusion. 
Therefore, from biological consideration, we study model (3) in the closed set

$$
\Gamma=\left\{(S, I, R) \in \mathbb{R}_{+}^{3}: 0<S+I+R \leq \frac{b}{d}\right\} .
$$

Proposition 2 is proved in [15] and is here just recalled.

Proposition 2. (i) The disease-free equilibrium $E_{0}=(b / d, 0$, $0)$ is globally asymptotically stable if $R_{0}<1$ and unstable if $R_{0}>1$ in the set $\Gamma$.

(ii) The endemic equilibrium $E^{*}=\left(S^{*}, I^{*}, R^{*}\right)$ of model (3) is locally asymptotically stable if $R_{0}>1$ in the set $\Gamma$.

Next, we present the following theorem which gives condition for the global asymptotical stability of the endemic equilibrium $E^{*}$ of model (3).

Theorem 3. If $R_{0}>1$, the endemic equilibrium $E^{*}=\left(S^{*}, I^{*}\right.$, $\left.R^{*}\right)$ of model (3) is globally asymptotically stable in the set $\Gamma$.

Proof. By summing all the equations of model (3), we find that the total population size verify the following equation:

$$
\frac{d N}{d t}=b-d N-\delta I
$$

where $N=S+I+R$.

It is convenient to choose the variable $(N, I, R)$ instead of $(S, I, R)$. That is, consider the following model:

$$
\begin{gathered}
\frac{d N}{d t}=b-d N-\delta I, \\
\frac{d I}{d t}=\left(\beta_{1}-\frac{\beta_{2} I}{m+I}\right)(N-I-R) I-(d+\mu+\delta) I, \\
\frac{d R}{d t}=\mu I-(d+\gamma) R,
\end{gathered}
$$

changing the variables such that $x=N-N^{*}, y=I-I^{*}$, and $z=R-R^{*}$, where $N^{*}=S^{*}+I^{*}+R^{*}$, so model (14) becomes as follows:

$$
\begin{aligned}
\frac{d x}{d t}= & -d x-\delta y, \\
\frac{d y}{d t}= & \left(\beta_{1}-\frac{\beta_{2} I^{*}}{m+I^{*}}\right) I^{*} \\
& \times\left(x-\left(1+\frac{m \beta_{2}(d+\mu+\delta)}{I^{*}\left(\beta_{1}-\beta_{2}\right)+\beta_{1} m}\right) y-z\right), \\
\frac{d z}{d t}= & \mu y-(d+\gamma) z .
\end{aligned}
$$

Consider the function

$$
V(x, y, z)=\frac{1}{2}\left(k_{1} x^{2}+y^{2}+k_{2} z^{2}\right)
$$

where $k_{1}$ and $k_{2}$ are positive constants which will be chosen later. Then the derivative of $V$ along the solution for model (15) is given by

$$
\begin{aligned}
\frac{d V}{d t}= & k_{1} x x_{t}+y y_{t}+k_{2} z z_{t} \\
= & k_{1} x(-d x-\delta y)+\left(\beta_{1}-\frac{\beta_{2} I^{*}}{m+I^{*}}\right) I^{*} y \\
& \times\left(x-\left(1+\frac{m \beta_{2}(d+\mu+\delta)}{I^{*}\left(\beta_{1}-\beta_{2}\right)+\beta_{1} m}\right) y-z\right) \\
& +k_{2} z(\mu y-(d+\gamma) z) \\
= & -d k_{1} x^{2}-\left(\beta_{1}-\frac{\beta_{2} I^{*}}{m+I^{*}}\right) \\
& \times\left(1+\frac{m \beta_{2}(d+\mu+\delta)}{I^{*}\left(\beta_{1}-\beta_{2}\right)+\beta_{1} m}\right) \\
& \times I^{*} y^{2}-k_{2}(d+\gamma) z^{2} \\
& +\left(\frac{\left(I^{*}\left(\beta_{1}-\beta_{2}\right)+\beta_{1} m\right) I^{*}}{m+I^{*}}-k_{1} \delta\right) x y \\
& +\left(k_{2} \mu-\frac{\left(I^{*}\left(\beta_{1}-\beta_{2}\right)+\beta_{1} m\right) I^{*}}{m+I^{*}}\right) y z .
\end{aligned}
$$

Let us choose $k_{1}$ and $k_{2}$ such that

$$
\begin{aligned}
& \frac{\left(I^{*}\left(\beta_{1}-\beta_{2}\right)+\beta_{1} m\right) I^{*}}{m+I^{*}}-k_{1} \delta=0, \\
& k_{2} \mu-\frac{\left(I^{*}\left(\beta_{1}-\beta_{2}\right)+\beta_{1} m\right) I^{*}}{m+I^{*}}=0 ;
\end{aligned}
$$

then $k_{1}=\left(I^{*}\left(\beta_{1}-\beta_{2}\right)+\beta_{1} m\right) I^{*} / \delta\left(m+I^{*}\right)$ and $k_{2}=\left(I^{*}\left(\beta_{1}-\right.\right.$ $\left.\left.\beta_{2}\right)+\beta_{1} m\right) I^{*} / \mu\left(m+I^{*}\right)$. Thus, we have

$$
\begin{aligned}
\frac{d V}{d t}= & -d k_{1} x^{2}-\left(\beta_{1}-\frac{\beta_{2} I^{*}}{m+I^{*}}\right) \\
& \times\left(1+\frac{m \beta_{2}(d+\mu+\delta)}{I^{*}\left(\beta_{1}-\beta_{2}\right)+\beta_{1} m}\right) I^{*} y^{2}-k_{2}(d+\gamma) z^{2} \leq 0 .
\end{aligned}
$$

By applying the Lyapunov-LaSalle asymptotic stability theorem $[41,42]$, the endemic equilibrium $E^{*}$ of model (3) is globally asymptotically stable. This completes the proof.

Example 4. We now use the parameter values

$$
\begin{array}{rlr}
b=5, \quad & d=0.02, \quad \beta_{1}=0.002, \quad & \beta_{2}=0.0018, \\
m=30, \quad \delta=0.1, \quad \mu=0.05, & \gamma=0.01
\end{array}
$$




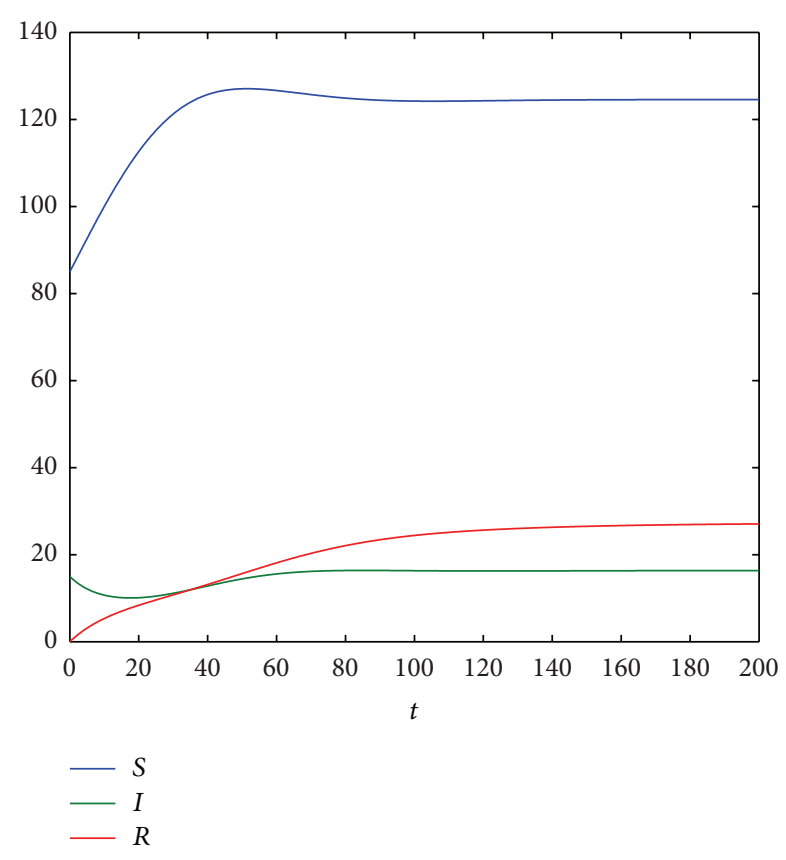

FIGURE 1: The global stability of the endemic equilibrium $E^{*}=$ $\left(S^{*}, I^{*}, R^{*}\right)$ for model (21) with initial values $S(0)=85, I(0)=15$, and $R(0)=0$. The parameters are taken as (20).

and show the stability of the endemic equilibrium $E^{*}$ of model (3). Model (3) becomes

$$
\begin{gathered}
\frac{d S}{d t}=5-0.02 S-\left(0.002-\frac{0.0018 I}{30+I}\right) S I+0.01 R \\
\frac{d I}{d t}=\left(0.002-\frac{0.0018 I}{30+I}\right) S I-(0.02+0.05+0.1) I \\
\frac{d R}{d t}=0.05 I-(0.02+0.01) R
\end{gathered}
$$

Note that

$$
R_{0}=\frac{b \beta_{1}}{d(d+\mu+\delta)}=2.941>1
$$

From Theorem 3, one can therefore conclude that, for any initial values $(S(0), I(0), R(0))$, the endemic equilibrium $E^{*}=$ $(124.564,16.361,27.269)$ of model $(21)$ is globally stable (see Figure 1).

\section{Stochastic Stability of the Endemic Equilibrium of Model (8)}

Throughout this paper, let $(\Omega, \mathscr{F}, \mathscr{P})$ be a complete probability space with a filtration $\left\{\mathscr{F}_{t}\right\}_{t \in \mathbb{R}_{+}}$satisfying the usual conditions (i.e., it is right continuous and increasing while $\mathscr{F}_{0}$ contains all $\mathscr{P}$-null sets).

Considering the general $n$-dimensional stochastic differential equation

$$
d x(t)=f(x(t), t) d t+\varphi(x(t), t) d B(t)
$$

on $t \geq 0$ with initial value $x(0)=x_{0}$, the solution is denoted by $x\left(t, x_{0}\right)$. Assume that $f(0, t)=0$ and $\varphi(0, t)=0$ for all $t \geq 0$, so (23) has the solution $x(t)=0$. This solution is called the trivial solution.

Definition 5 ( see [43]). The trivial solution $x(t)=0$ of (23) is said to be as follows:

(i) stable in probability if for all $\varepsilon>0$,

$$
\lim _{x_{0} \rightarrow 0} \mathscr{P}\left(\sup _{t \geq 0}\left|x\left(t, x_{0}\right)\right| \geq \varepsilon\right)=0
$$

(ii) asymptotically stable if it is stable in probability and, moreover,

$$
\lim _{x_{0} \rightarrow 0} \mathscr{P}\left(\lim _{t \rightarrow \infty} x\left(t, x_{0}\right)=0\right)=1
$$

(iii) asymptotically stable in the large if it is stable in probability and, moreover, for all $x_{0} \in \mathbb{R}^{n}$

$$
\mathscr{P}\left(\lim _{t \rightarrow \infty} x\left(t, x_{0}\right)=0\right)=1
$$

Define the differential operator $L$ associated to (23) by

$$
L=\frac{\partial}{\partial t}+\sum_{i=1}^{n} f_{i}(x, t) \frac{\partial}{\partial x_{i}}+\frac{1}{2} \sum_{i, j=1}^{n}\left[\varphi^{T}(x, t) \varphi(x, t)\right]_{i j} \frac{\partial^{2}}{\partial x_{i} \partial x_{j}} .
$$

If $L$ acts on a function $V(x, t) \in C^{2,1}\left(\mathbb{R}^{d} \times(0, \infty) ; \mathbb{R}_{+}\right)$, then

$$
\begin{aligned}
L V(x, t)= & V_{t}(x, t)+V_{x}(x, t) f(x, t) \\
& +\frac{1}{2} \operatorname{trace}\left[\varphi^{T}(x, t) V_{x x}(x, t) \varphi(x, t)\right],
\end{aligned}
$$

where $T$ means transposition. For more definitions of stability we refer to [43].

In the following, we will give the result of the asymptotical stability in the large of the endemic equilibrium $E^{*}$ of model (8).

If $R_{0}>1$, stochastic model (8) can center at its endemic equilibrium $E^{*}$. By the change of variables

$$
u=S-S^{*}, \quad v=I-I^{*}, \quad w=R-R^{*},
$$

we obtain the following system:

$$
d z(t)=f_{1}(z(t)) d t+f_{2}(z(t)) d B(t)
$$

where 


$$
f_{1}(z(t))=\left(\begin{array}{c}
z(t)=(u(t), v(t), w(t))^{T}, \\
-\left(d+\left(\beta_{1}-\frac{\beta_{2} I^{*}}{I^{*}+m}\right) I^{*}\right) u-\left(d+\mu+\delta-\frac{\beta_{2} m S^{*} I^{*}}{\left(I^{*}+m\right)^{2}}\right) v+\gamma w \\
\left(\beta_{1}-\frac{\beta_{2} I^{*}}{I^{*}+m}\right) I^{*} u-\frac{\beta_{2} m S^{*} I^{*}}{\left(I^{*}+m\right)^{2}} v \\
\mu v-(d+\gamma) w \\
f_{2}(z(t))=\left(\begin{array}{ccc}
\sigma_{1} u(t) & 0 & 0 \\
0 & \sigma_{2} v(t) & 0 \\
0 & 0 & \sigma_{3} w(t)
\end{array}\right) .
\end{array}\right.
$$

It is easy to see that the stability of the endemic equilibrium $E^{*}$ of model (8) is equivalent to the stability of the trivial solution for model (30).

Before proving the stochastic stability of the trivial solution for model (30), we put forward a Lemma in [44].

Lemma 6 (see [44]). Suppose that there exists a function $V(z, t) \in C^{2}(\Omega)$ satisfying the following inequalities:

$$
\begin{gathered}
K_{1}|z|^{\omega} \leq V(z, t) \leq K_{2}|z|^{\omega}, \\
L V(z, t) \leq-K_{3}|z|^{\omega},
\end{gathered}
$$

where $\omega>0$ and $K_{i}(i=1,2,3)$ is positive constant. Then the trivial solution for model (30) is exponentially $\omega$-stable for all time $t \geq 0$. When $\omega=2$, it is usually said to be exponentially stable in mean square and the trivial solution $x=0$ is asymptotically stable in the large.

From the above Lemma, we obtain the following theorem.

Theorem 7. Assume that $R_{0}=b \beta_{1} / d(d+\mu+\delta)>1$. If the following conditions are satisfied:

$$
\begin{gathered}
\sigma_{1}^{2}<2 d, \quad \sigma_{3}^{2}<2(d+\gamma), \\
\frac{2\left(\gamma^{2}+\mu^{2}\right)}{2(d+\gamma)-\sigma_{3}^{2}}<d+\mu+\delta+\frac{\beta_{2} m \theta S^{*} I^{*}}{\left(I^{*}+m\right)^{2}} \\
\sigma_{2}^{2}<\frac{2}{1+\theta}\left(d+\mu+\delta+\frac{\beta_{2} m \theta S^{*} I^{*}}{\left(I^{*}+m\right)^{2}}-\frac{2\left(\gamma^{2}+\mu^{2}\right)}{2(d+\gamma)-\sigma_{3}^{2}}\right),
\end{gathered}
$$

where

$$
\theta=\frac{(2 d+\mu+\delta)\left(I^{*}+m\right)}{\left(\left(\beta_{1}-\beta_{2}\right) I^{*}+\beta_{1} m\right) I^{*}},
$$

then the trivial solution of model (30) is asymptotically stable in the large. And the endemic point $E^{*}$ of model (8) is asymptotically stable in the large.
Proof. We define the Lyapunov function $V(u, v, w)$ as follows:

$$
\begin{aligned}
V(z(t)) & =\frac{1}{2} c_{1}(u+v)^{2}+\frac{1}{2} c_{2} v^{2}+\frac{1}{2} c_{3} w^{2} \\
& :=V_{1}(z(t))+V_{2}(z(t))+V_{3}(z(t)),
\end{aligned}
$$

where $c_{1}>0, c_{2}>0$ and $c_{3}>0$ are real positive constants to be chosen later. It is easy to check that inequalities (32) are true.

Furthermore, by the Itô formula, we have

$$
\begin{aligned}
L V_{1}= & c_{1}(u+v)(-d u-(d+\mu+\delta) v+\gamma w) \\
& +\frac{1}{2} c_{1} \sigma_{1}^{2} u^{2}+\frac{1}{2} c_{1} \sigma_{2}^{2} v^{2} \\
= & -c_{1}\left(d-\frac{1}{2} \sigma_{1}^{2}\right) u^{2}-c_{1}(2 d+\mu+\delta) u v \\
& -c_{1}\left(d+\mu+\delta-\frac{1}{2} \sigma_{2}^{2}\right) v^{2}+c_{1} \gamma u w+c_{1} \gamma v w \\
L V_{2}= & c_{2} v\left(\left(\beta_{1}-\frac{\beta_{2} I^{*}}{I^{*}+m}\right) I^{*} u-\frac{\beta_{2} m S^{*} I^{*}}{\left(I^{*}+m\right)^{2}} v\right) \\
& +\frac{1}{2} c_{2} \sigma_{2}^{2} v^{2} \\
= & c_{2}\left(\beta_{1}-\frac{\beta_{2} I^{*}}{I^{*}+m}\right) I^{*} u v-c_{2}\left(\frac{\beta_{2} m S^{*} I^{*}}{\left(I^{*}+m\right)^{2}}-\frac{1}{2} \sigma_{2}^{2}\right) v^{2} \\
L V_{3}= & c_{3} w(\mu v-(d+\gamma) w)+\frac{1}{2} c_{3} \sigma_{3}^{2} w^{2} \\
= & c_{3} \mu v w-c_{3}\left(d+\gamma-\frac{1}{2} \sigma_{3}^{2}\right) w^{2}
\end{aligned}
$$

Then we have

$$
\begin{aligned}
L V & =L V_{1}+L V_{2}+L V_{3} \\
& =-c_{1}\left(d-\frac{1}{2} \sigma_{1}^{2}\right) u^{2}
\end{aligned}
$$




$$
\begin{aligned}
& -\left(c_{1}(d+\mu+\delta)+\frac{c_{2} \beta_{2} m S^{*} I^{*}}{\left(I^{*}+m\right)^{2}}\right. \\
& \left.\quad-\frac{1}{2} \sigma_{2}^{2}\left(c_{1}+c_{2}\right)\right) v^{2} \\
& -c_{2}\left(d+\gamma-\frac{1}{2} \sigma_{3}^{2}\right) w^{2} \\
& -\left(c_{1}(2 d+\mu+\delta)-c_{2}\left(\beta_{1}-\frac{\beta_{2} I^{*}}{I^{*}+m}\right) I^{*}\right) u v \\
& +c_{1} \gamma u w+c_{3} \mu v w+c_{1} \gamma v w .
\end{aligned}
$$

Choose $c_{3}=c_{1}$ and

$$
c_{1}(2 d+\mu+\delta)-c_{2}\left(\beta_{1}-\frac{\beta_{2} I^{*}}{I^{*}+m}\right) I^{*}=0
$$

then

$$
c_{2}=\frac{c_{1}(2 d+\mu+\delta)\left(I^{*}+m\right)}{\left(\left(\beta_{1}-\beta_{2}\right) I^{*}+\beta_{1} m\right) I^{*}}=c_{1} \theta .
$$

Moreover, using Cauchy inequality to $\gamma u w, \gamma \nu w$, and $\mu \nu w$, we can obtain

$$
\begin{aligned}
& \gamma u w \leq \frac{\gamma^{2} u^{2}}{d+\gamma-(1 / 2) \sigma_{3}^{2}}+\frac{1}{4}\left(d+\gamma-\frac{1}{2} \sigma_{3}^{2}\right) w^{2}, \\
& \mu v w \leq \frac{\mu^{2} v^{2}}{d+\gamma-(1 / 2) \sigma_{3}^{2}}+\frac{1}{4}\left(d+\gamma-\frac{1}{2} \sigma_{3}^{2}\right) w^{2}, \\
& \gamma v w \leq \frac{\gamma^{2} v^{2}}{d+\gamma-(1 / 2) \sigma_{3}^{2}}+\frac{1}{4}\left(d+\gamma-\frac{1}{2} \sigma_{3}^{2}\right) w^{2} .
\end{aligned}
$$

Substituting (39) and (40) into (37), yields

$$
\begin{aligned}
L V= & -\left(d-\frac{1}{2} \sigma_{1}^{2}-\frac{c_{1} \gamma^{2}}{d+\gamma-(1 / 2) \sigma_{3}^{2}}\right) u^{2} \\
& -\frac{1}{4}\left(3 c_{3}-2 c_{1}\right)\left(d+\gamma-\frac{1}{2} \sigma_{3}^{2}\right) w^{2} \\
& -\left(c_{1}(d+\mu+\delta)+\frac{c_{2} \beta_{2} m S^{*} I^{*}}{\left(I^{*}+m\right)^{2}}-\frac{1}{2} \sigma_{2}^{2}\left(c_{1}+c_{2}\right)\right. \\
= & \left.-\frac{c_{1} \gamma^{2}+c_{3} \mu^{2}}{d+\gamma-(1 / 2) \sigma_{3}^{2}}\right) v^{2} \\
= & \left(A u^{2}+B v^{2}+C w^{2}\right),
\end{aligned}
$$

where

$$
\begin{gathered}
A=d-\frac{1}{2} \sigma_{1}^{2}-\frac{c_{1} \gamma^{2}}{d+\gamma-(1 / 2) \sigma_{3}^{2}}, \\
B=c_{1}\left(d+\mu+\delta+\frac{\beta_{2} m \theta S^{*} I^{*}}{\left(I^{*}+m\right)^{2}}-\frac{1}{2} \sigma_{2}^{2}(1+\theta)\right. \\
\left.-\frac{\gamma^{2}+\mu^{2}}{d+\gamma-(1 / 2) \sigma_{3}^{2}}\right) \\
C=\frac{1}{4} c_{1}\left(d+\gamma-\frac{1}{2} \sigma_{3}^{2}\right) .
\end{gathered}
$$

Let us choose $c_{1}$ such that

$$
0<c_{1}<\frac{1}{\gamma^{2}}\left(d-\frac{1}{2} \sigma_{1}^{2}\right)\left(d+\gamma-\frac{1}{2} \sigma_{3}^{2}\right) .
$$

On the other hand, the conditions in (33) are satisfied, so $A, B$, and $C$ are positive constants. Let $\lambda=\min \{A, B, C\}$; then $\lambda>0$. From (41), one sees that

$$
L V(z(t)) \leq-\lambda|z(t)|^{2}
$$

According to Lemma 6, we therefore conclude that the trivial solution of model (30) is asymptotically stable in the large. We therefore have the assertion.

Next, for further studying the effects of noise on the dynamics of model (8), we give some numerical examples to illustrate the dynamical behavior of stochastic model (8) by using the Milstein method mentioned in Higham [45]. In this way, model (8) can be rewritten as the following discretization equations:

$$
\begin{aligned}
S_{k+1}= & S_{k}+\left(b-d S_{k}-\left(\beta_{1}-\frac{\beta_{2} I_{k}}{m+I_{k}}\right) S_{k} I_{k}+\gamma R_{k}\right) \\
& \times \Delta t+\sigma_{1}\left(S_{k}-S^{*}\right) \sqrt{\Delta t} \xi_{k}, \\
I_{k+1}= & I_{k}+\left(\left(\beta_{1}-\frac{\beta_{2} I_{k}}{m+I_{k}}\right) S_{k} I_{k}-(d+\mu+\delta) I_{k}\right) \\
& \times \Delta t+\sigma_{2}\left(I_{k}-I^{*}\right) \sqrt{\Delta t} \eta_{k}, \\
R_{k+1}= & R_{k}+\left(\mu I_{k}-(d+\gamma) R_{k}\right) \\
& \times \Delta t+\sigma_{3}\left(R_{k}-R^{*}\right) \sqrt{\Delta t} \zeta_{k},
\end{aligned}
$$

where $\xi_{k}, \zeta_{k}$, and $\eta_{k}, k=1,2, \ldots, n$, are the Gaussian random variables $N(0,1)$. 


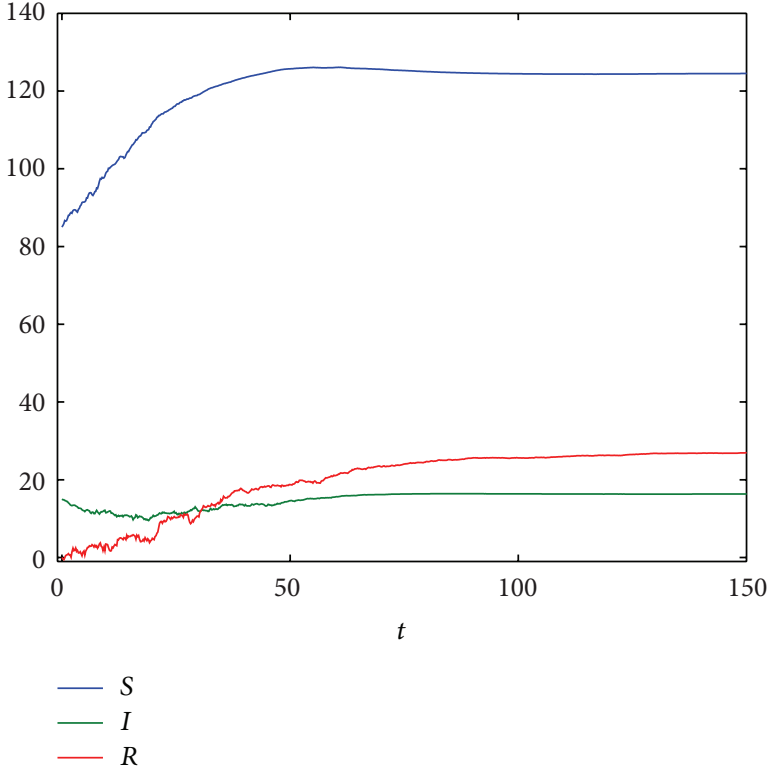

(a) $\sigma_{1}=0.025, \sigma_{2}=0.1, \sigma_{3}=0.05$

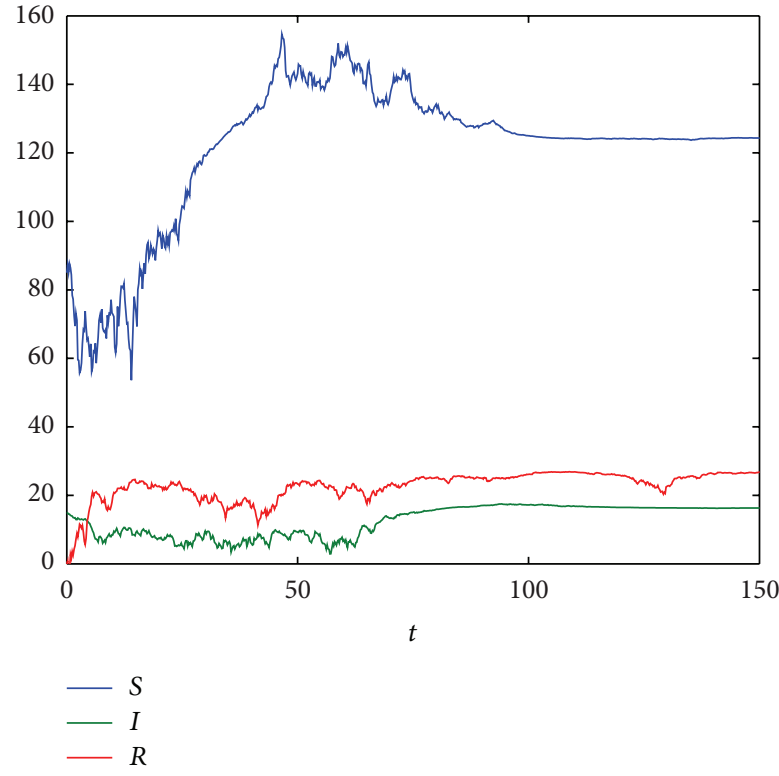

(b) $\sigma_{1}=0.18, \sigma_{2}=0.18, \sigma_{3}=0.22$

Figure 2: The asymptotic behavior of the solutions to the stochastic model (47) around the endemic equilibrium $E^{*}$ with initial values $S(0)=85, I(0)=15$, and $R(0)=0$. The parameters are taken as $(20)$.

The parameters of model (8) are fixed as (20). Then model (8) has the endemic point $E^{*}=(124.564,16.361,27.269)$. And model (8) becomes

$$
\begin{aligned}
d S= & 5-0.02 S-\left(0.002-\frac{0.0018 I}{30+I}\right) S I \\
& +0.01 R+\sigma_{1}(S-124.564) d B_{1}(t), \\
d I= & \left(0.002-\frac{0.0018 I}{30+I}\right) S I-(0.02+0.05+0.1) I \\
& +\sigma_{2}(I-16.361) d B_{2}(t), \\
d R= & 0.05 I-(0.02+0.01) R \\
& +\sigma_{3}(R-27.269) d B_{3}(t) .
\end{aligned}
$$

Choosing $\left(\sigma_{1}, \sigma_{2}, \sigma_{3}\right)=(0.025,0.1,0.05)$ and noting that

$$
\begin{gathered}
R_{0}=\frac{b \beta_{1}}{d(d+\mu+\delta)}=2.941>1, \\
\sigma_{1}^{2}=0.025^{2}<2 d=0.04 \\
\sigma_{3}^{2}=0.05^{2}<2(d+\gamma)=0.06 \\
\sigma_{2}^{2}=0.1^{2}<\frac{2}{1+\theta}\left(d+\mu+\delta+\frac{\beta_{2} m \theta S^{*} I^{*}}{\left(I^{*}+m\right)^{2}}-\frac{2\left(\gamma^{2}+\mu^{2}\right)}{2(d+\gamma)-\sigma_{3}^{2}}\right) \\
=0.12739-\frac{0.0011}{0.06-0.05^{2}}=0.108 .
\end{gathered}
$$

It is easy to see that all the conditions of Theorem 7 are satisfied, and we can therefore conclude that the endemic point $E^{*}$ of model (47) is asymptotically stable in the large. The numerical examples shown in Figure 2(b) clearly support these results. To further illustrate the effect of the noise intensity on model (47), we keep all the parameters in (20) unchanged but increase $\left(\sigma_{1}, \sigma_{2}, \sigma_{3}\right)$ to $(0.18,0.18,0.22)$. In this case,

$$
\begin{gathered}
\sigma_{1}^{2}=0.0324<0.04, \quad \sigma_{3}^{2}=0.0484<0.06 \\
\sigma_{2}^{2}=0.0324<0.12739-\frac{0.0011}{0.06-0.22^{2}}=0.033 ;
\end{gathered}
$$

we can therefore conclude, by Theorem 7 , that for any initial value ( $S(0), I(0), R(0)$ ), the endemic point $E^{*}$ of model (47) is asymptotically stable in the large (see Figure 2(b)).

In the above case, if we adopt $d=0.01$ and keep the other parameters unchanged, in this case, model (47) has the endemic point $E^{*}=(138.935,26.746,66.864)$. And it is easy to compute

$$
\begin{gathered}
R_{0}=\frac{b \beta_{1}}{d(d+\mu+\delta)}=6.25>1, \\
\sigma_{1}^{2}=0.0324>2 d=0.02, \\
\sigma_{3}^{2}=0.0484>2(d+\gamma)=0.04 .
\end{gathered}
$$

Therefore, the conditions of Theorem 7 are not satisfied, and the solution of model (47) will oscillate strongly around the endemic point $E^{*}=(138.935,26.746,66.864)$, which is not asymptotically stable in the large (see Figure 3 ). 


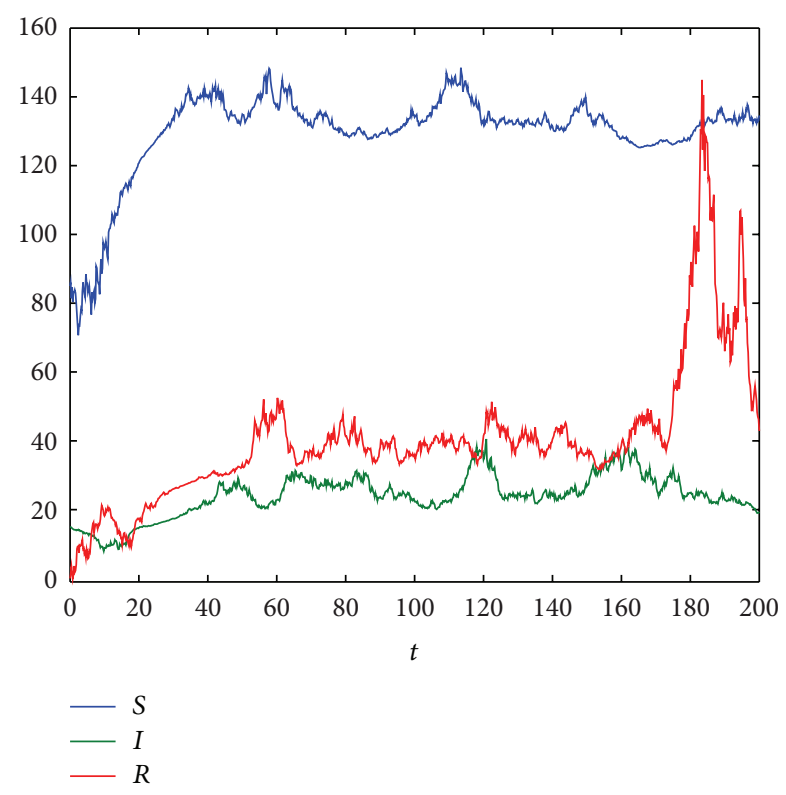

FIgURE 3: The global stability of the endemic equilibrium $E^{*}=$ $\left(S^{*}, I^{*}, R^{*}\right)$ for model (47) with initial values $S(0)=85, I(0)=15$, and $R(0)=0$. The parameters are taken as $b=5, d=0.01$, $\beta_{1}=0.002, \beta_{2}=0.0018, m=30, \delta=0.1, \mu=0.05, \gamma=0.01$, $\sigma_{1}=0.18, \sigma_{2}=0.18$, and $\sigma_{3}=0.22$.

\section{Conclusions and Discussions}

In this paper, by using the theory of stochastic differential equation, we investigate the dynamics of a SIRS epidemic model incorporating media coverage with random perturbation. The value of this study lies in two aspects. First, it presents some relevant properties of the deterministic model (3), including boundedness and the stability of the diseasefree and endemic points. Second, it verifies the stochastic stability in the large of the endemic equilibrium for the stochastic model (8).

From the theoretical and numerical results, we can know that, when the noise density is not large, the stochastic model (8) preserves the property of the stability of the deterministic model (3). To a great extent, we can ignore the noise and use the deterministic model (3) to describe the population dynamics. However, when the noise is sufficiently large, it can force the population to become largely fluctuating. In this case, we can not use deterministic model (3) but instead stochastic model (8) to describe the population dynamics. Needless to say, both deterministic and stochastic epidemic models have their important roles.

\section{Acknowledgments}

The author would like to thank the editors and referees for their helpful comments and suggestions. This research was supported by the National Science Foundation of China (61272018 \& 61373005) and Zhejiang Provincial Natural Science Foundation (R1110261).

\section{References}

[1] Z. Ma, Y. Zhou, and J. Wu, Modeling and Dynamics of Infectious Diseases, Higher Education Press, Beijing, China, 2009.

[2] A. Korobeinikov and P. K. Maini, "Non-linear incidence and stability of infectious disease models," Mathematical Medicine and Biology, vol. 22, no. 2, pp. 113-128, 2005.

[3] V. Capasso and G. Serio, "A generalization of the KermackMcKendrick deterministic epidemic model," Mathematical Biosciences, vol. 42, no. 1-2, pp. 43-61, 1978.

[4] W.-M. Liu, S. A. Levin, and Y. Iwasa, "Influence of nonlinear incidence rates upon the behavior of SIRS epidemiological models," Journal of Mathematical Biology, vol. 23, no. 2, pp. 187204, 1985.

[5] W.-M. Liu, H. W. Hethcote, and S. A. Levin, "Dynamical behavior of epidemiological models with nonlinear incidence rates," Journal of Mathematical Biology, vol. 25, no. 4, pp. 359$380,1987$.

[6] H. W. Hethcote, "Mathematics of infectious diseases," SIAM Review, vol. 42, no. 4, pp. 599-653, 2000.

[7] S. Ruan and W. Wang, "Dynamical behavior of an epidemic model with a nonlinear incidence rate," Journal of Differential Equations, vol. 188, no. 1, pp. 135-163, 2003.

[8] D. Xiao and S. Ruan, "Global analysis of an epidemic model with nonmonotone incidence rate," Mathematical Biosciences, vol. 208, no. 2, pp. 419-429, 2007.

[9] Y. Xiao and S. Tang, "Dynamics of infection with nonlinear incidence in a simple vaccination model," Nonlinear Analysis: Real World Applications, vol. 11, no. 5, pp. 4154-4163, 2010.

[10] Y. Xiao, X. Xu, and S. Tang, "Sliding mode control of outbreaks of emerging infectious diseases," Bulletin of Mathematical Biology, vol. 74, no. 10, pp. 2403-2422, 2012.

[11] J. Zhang, Z. Jin, G.-Q. Sun, T. Zhou, and S. Ruan, "Analysis of rabies in China: transmission dynamics and control," PloS One, vol. 6, no. 7, article e20891, 2011.

[12] J. Cui, Y. Sun, and H. Zhu, "The impact of media on the control of infectious diseases," Journal of Dynamics and Differential Equations, vol. 20, no. 1, pp. 31-53, 2008.

[13] J.-A. Cui, X. Tao, and H. Zhu, "An SIS infection model incorporating media coverage," Rocky Mountain Journal of Mathematics, vol. 38, no. 5, pp. 1323-1334, 2008.

[14] R. Liu, J. Wu, and H. Zhu, "Media/psychological impact on multiple outbreaks of emerging infectious diseases," Computational and Mathematical Methods in Medicine, vol. 8, no. 3, pp. 153164, 2007.

[15] Y. Liu and J.-A. Cui, "The impact of media coverage on the dynamics of infectious disease," International Journal of Biomathematics, vol. 1, no. 1, pp. 65-74, 2008.

[16] C. Sun, W. Yang, J. Arino, and K. Khan, "Effect of mediainduced social distancing on disease transmission in a two patch setting," Mathematical Biosciences, vol. 230, no. 2, pp. 87-95, 2011.

[17] J. M. Tchuenche, N. Dube, C. P. Bhunu, R. J. Smith, and C. T. Bauch, "The impact of media coverage on the transmission dynamics of human influenza," BMC Public Health, vol. 11, no. 1, article S5, 2011.

[18] Y. Xiao, T. Zhao, and S. Tang, "Dynamics of an infectious diseases with media/psychology induced non-smooth incidence," Mathematical Biosciences and Engineering, vol. 10, no. 2, pp. 445-461, 2013. 
[19] J. R. Beddington and R. M. May, "Harvesting natural populations in a randomly fluctuating environment," Science, vol. 197, no. 4302, pp. 463-465, 1977.

[20] L. J. Allen, "An introduction to stochastic epidemic models," in Mathematical Epidemiology, F. Brauer, P. van den Driessche, and J. Wu, Eds., pp. 81-130, Springer, Berlin, Germany, 2008.

[21] T. C. Gard, Introduction to Stochastic Differential Equations, Dekker, New York, NY, USA, 1988.

[22] B. Øksendal, Stochastic Differential Equations: An Introduction with Applications, Springer, 1995.

[23] X. Mao, Stochastic Differential Equations and Their Applications, Horwood, 1997.

[24] E. Beretta, V. Kolmanovskii, and L. Shaikhet, "Stability of epidemic model with time delays influenced by stochastic perturbations," Mathematics and Computers in Simulation, vol. 45, no. 3-4, pp. 269-277, 1998.

[25] R. Z. Khasminskii and F. C. Klebaner, "Long term behavior of solutions of the lotka-volterra system under small random perturbations," Annals of Applied Probability, vol. 11, no. 3, pp. 952-963, 2001.

[26] X. Mao, G. Marion, and E. Renshaw, "Environmental Brownian noise suppresses explosions in population dynamics," Stochastic Processes and Their Applications, vol. 97, no. 1, pp. 95-110, 2002.

[27] I. Nåsell, "Stochastic models of some endemic infections," Mathematical Biosciences, vol. 179, no. 1, pp. 1-19, 2002.

[28] E. Tornatore, S. M. Buccellato, and P. Vetro, "Stability of a stochastic SIR system," Physica A, vol. 354, no. 1-4, pp. 111-126, 2005.

[29] Q. Luo and X. Mao, "Stochastic population dynamics under regime switching," Journal of Mathematical Analysis and Applications, vol. 334, no. 1, pp. 69-84, 2007.

[30] M. Liu and K. Wang, "Survival analysis of stochastic singlespecies population models in polluted environments," Ecological Modelling, vol. 220, no. 9-10, pp. 1347-1357, 2009.

[31] J. Yu, D. Jiang, and N. Shi, "Global stability of two-group SIR model with random perturbation," Journal of Mathematical Analysis and Applications, vol. 360, no. 1, pp. 235-244, 2009.

[32] T. Britton, "Stochastic epidemic models: a survey," Mathematical Biosciences, vol. 225, no. 1, pp. 24-35, 2010.

[33] F. Ball, D. Sirl, and P. Trapman, "Analysis of a stochastic SIR epidemic on a random network incorporating household structure," Mathematical Biosciences, vol. 224, no. 2, pp. 53-73, 2010.

[34] D. Jiang, C. Ji, N. Shi, and J. Yu, "The long time behavior of DI SIR epidemic model with stochastic perturbation," Journal of Mathematical Analysis and Applications, vol. 372, no. 1, pp. 162-180, 2010.

[35] D. Jiang, J. Yu, C. Ji, and N. Shi, "Asymptotic behavior of global positive solution to a stochastic SIR model," Mathematical and Computer Modelling, vol. 54, no. 1-2, pp. 221-232, 2011.

[36] J. Lv and K. Wang, "Asymptotic properties of a stochastic predator-prey system with Holling II functional response," Communications in Nonlinear Science and Numerical Simulation, vol. 16, no. 10, pp. 4037-4048, 2011.

[37] A. Gray, D. Greenhalgh, L. Hu, X. Mao, and J. Pan, "A stochastic differential equation SIS epidemic model," SIAM Journal on Applied Mathematics, vol. 71, no. 3, pp. 876-902, 2011.

[38] X. Wang, H. Huang, Y. Cai, and W. Wang, "The complex dynamics of a stochastic predatorprey model," Abstract and Applied Analysis, vol. 2012, Article ID 401031, 24 pages, 2012.
[39] Y. Cai, X. Wang, W. Wang, and M. Zhao, "Stochastic dynamics of a SIRS epidemic model with ratio-dependent incidence rate," Abstract and Applied Analysis, vol. 2013, Article ID 172631, 11 pages, 2013.

[40] Z. Liu, "Dynamics of positive solutions to SIR and SEIR epidemic models with saturated incidence rates," Nonlinear Analysis: Real World Applications, vol. 14, no. 3, pp. 1286-1299, 2013.

[41] J. P. LaSalle, “The stability of dynamical systems," Society for Industrial and Applied Mathematics, vol. 25, 1987.

[42] A. M. Lyapunov, "The general problem of the stability of motion," International Journal of Control, vol. 55, no. 3, pp. 531534, 1992.

[43] R. Z. Khas'minskiü, Stochastic Stability of Differential Equations, vol. 7, Kluwer Academic Publishers, 1980.

[44] V. N. Afanasiev, V. B. Kolmanovskiı̌, and V. R. Nosov, Mathematical Theory of Control Systems Design, Kluwer Academic Publishers, 1996.

[45] D. J. Higham, "An algorithmic introduction to numerical simulation of stochastic differential equations," SIAM Review, vol. 43, no. 3, pp. 525-546, 2001. 


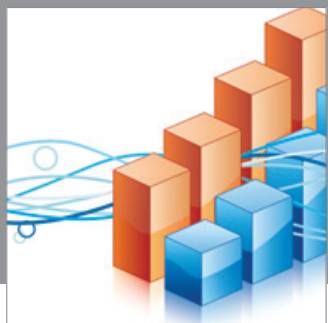

Advances in

Operations Research

mansans

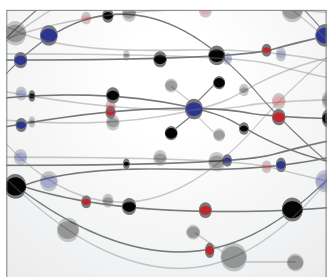

The Scientific World Journal
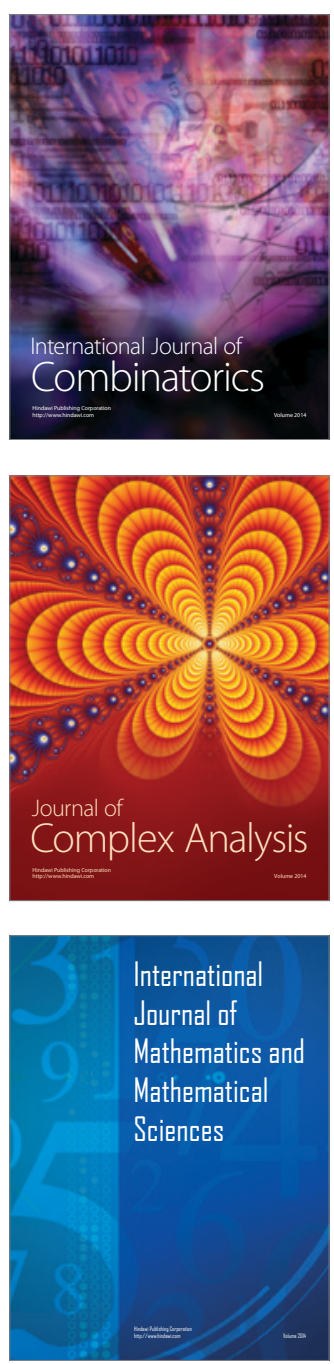
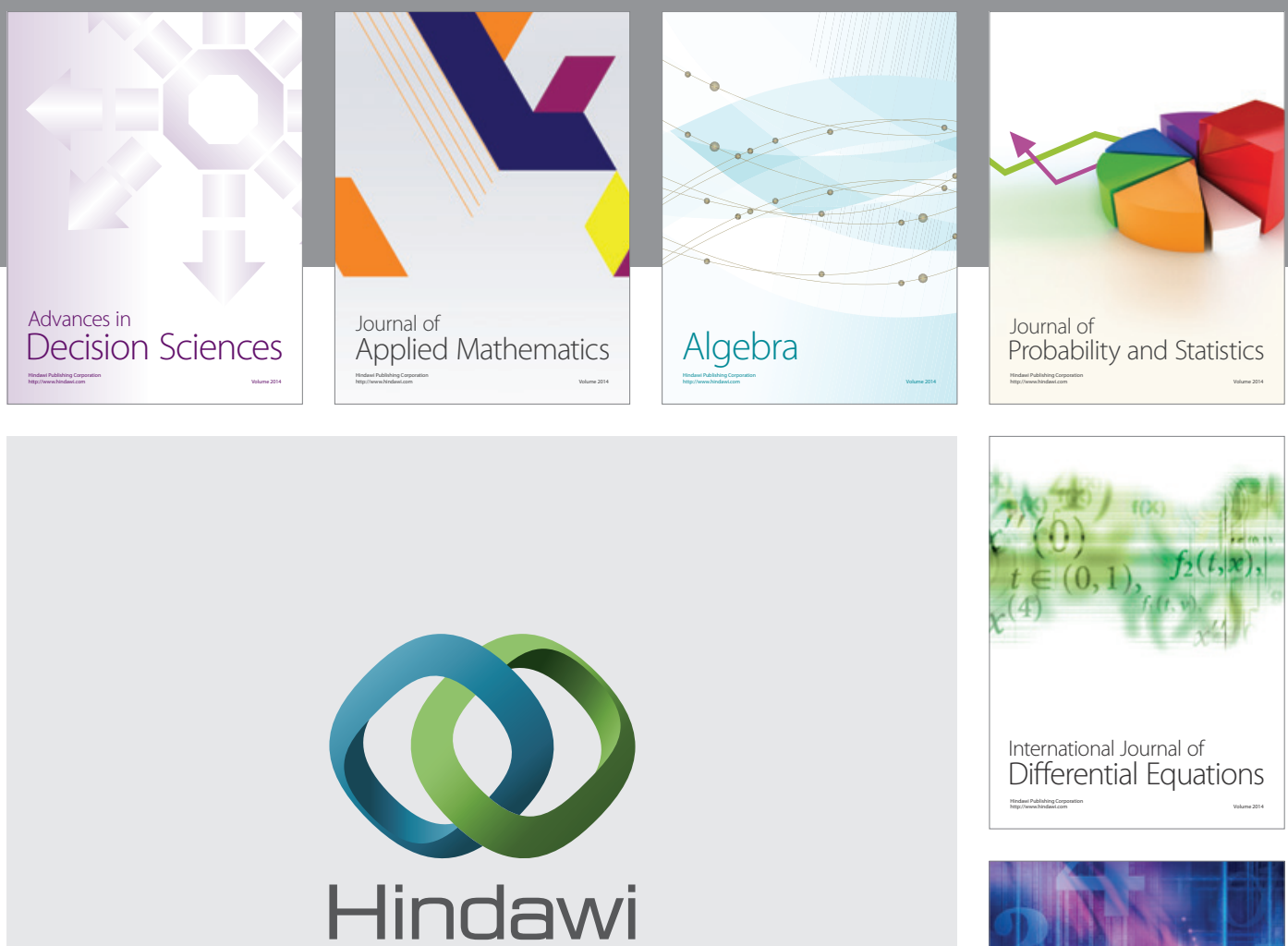

Submit your manuscripts at http://www.hindawi.com
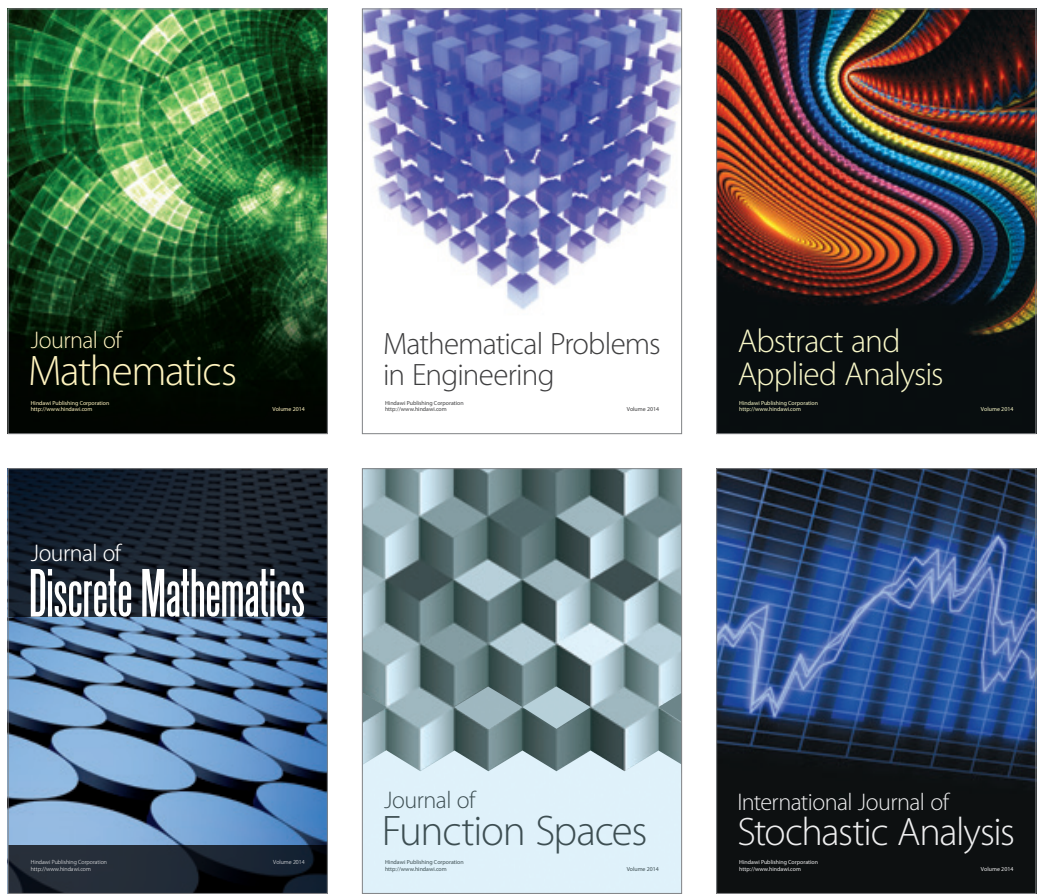

Journal of

Function Spaces

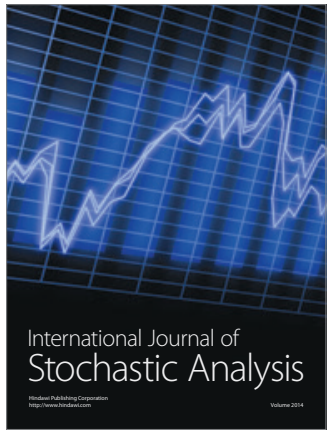

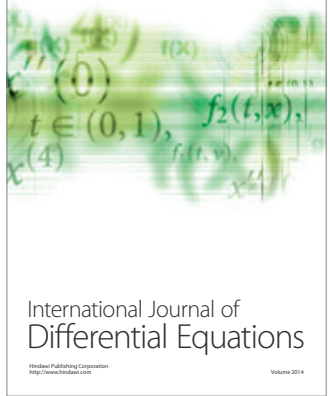
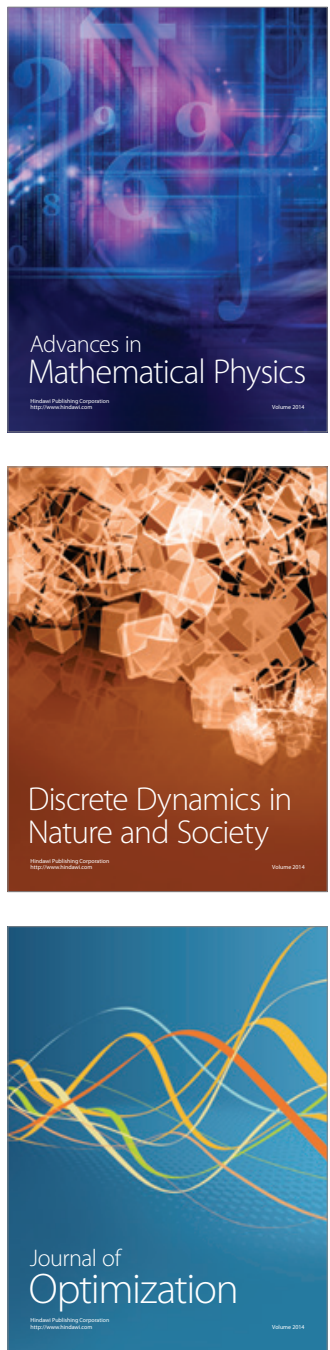